\title{
The In-silico Studies of Benzylidene Indanone Derivatives Towards Dengue Virus Type-2 NS2B/NS3 Protease
}

\author{
Nadirah Zawani Mohd Nesfu, ${ }^{1,2}$ Dominique Laurain-Mattar, ${ }^{2}$ \\ Ezatul E. Kamarulzaman, ${ }^{3}$ Habibah A. Wahab, ${ }^{3}$ Iffah Izzati Zakaria, ${ }^{4,5}$ \\ Mohd Zaheen Hassan, ${ }^{6}$ Nicolas Brosse, ${ }^{7}$ and Hasnah Osman ${ }^{1 *}$ \\ ${ }^{1}$ School of Chemical Sciences, Universiti Sains Malaysia, \\ 11800 USM Pulau Pinang, Malaysia \\ ${ }^{2}$ CNRS, L2CM, Faculté des Sciences et Technologies BP 70239, \\ Université de Lorraine, 54506 Vandoeuvre-lès-Nancy, France \\ ${ }^{3}$ School of Pharmaceutical Sciences, Universiti Sains Malaysia, \\ 11800 USM Pulau Pinang, Malaysia \\ ${ }^{4}$ Malaysian Institute of Pharmaceutical and Nutraceutical (IPharm), \\ Block 5-A, Halaman Bukit Gambir, 11700 Pulau Pinang, Malaysia \\ ${ }^{5}$ Malaysia Genome Institute, National Institutes of Biotechnology Malaysia, \\ 43000 Kajang, Selangor, Malaysia \\ ${ }^{6}$ College of Pharmacy, King Khalid University, 61421, Abha, Saudi Arabia \\ ${ }^{7}$ LERMAB, Faculté des Sciences et Technologies BP 70239, \\ Université de Lorraine, 54506 Vandoeuvre-lès-Nancy, France \\ *Corresponding author: ohasnah@usm.my
}

Published online: 25 December 2019

To cite this article: Mohd Nesfu, N. Z. et al. (2019). The in-silico studies of benzylidene indanone derivatives towards dengue virus type-2 NS2B/NS3 protease. J. Phys. Sci., 30(Supp. 2), 191-198, https://doi.org/10.21315/jps2019.30.s2.16

To link to this article: https://doi.org/10.21315/jps2019.30.s2.16

\begin{abstract}
This research focuses on the in-silico study of ten synthesised indanone derivatives as dengue virus type-2 (DENV-2) NS2B/NS3 protease inhibitor using Wilchapong et al.'s homology protein crystal structure. The effort to prevent the infection and cure the disease were escalating as the dengue virus transmission has been classified as an emerging infectious disease. The Lamarckian genetic algorithm was employed in Autodock 4.2 to determine the binding modes and synthesised compounds conformation towards DENV-2 NS2B/NS3 protease homology protein crystal structure. The in-silico study reveals that the compound, $3 g$ and $3 h$ have the highest binding affinity and fit into the allosteric pocket of DENV-2 NS2B/NS3 serine protease with hydrogen bonding, the $\pi-\pi$ stacking interaction and hydrophobic interaction.
\end{abstract}

Keywords: in-silico, indanone derivatives, dengue, NS2B/NS3, protease 


\section{INTRODUCTION}

Dengue fever (DF), a mosquito-borne viral infection, is transmitted to humans through the bites of the infected female mosquitoes of Aedes aegypti and Aedes albopictus. Four dengue virus serotypes (DENV 1-4) have been classified. The treatment of DF available nowadays is limited to supplemental medical care and fluid replacement, as no specific treatment exists. ${ }^{1}$ The first tetravalent vaccine Dengvaxia ${ }^{\circledR}$ developed by Sanofi Pasteur was licensed and approved by regulatory authorities in 20 countries for trial use by person ranging 9-45 years in a highly endemic area. ${ }^{2}$ However, the trial outcome showed that the vaccine trial participants faced a higher risk of more severe DF and hospitalisations compared to unvaccinated participants. ${ }^{3}$ With the absence of promising anti-dengue drugs or vaccine options, there are ongoing needs to seek other preventing and treatment measure. Therefore, the quest in searching for anti-dengue drugs or dengue vaccine is still ongoing.

Several synthetic strategists have been developed inspired by the useful moiety of indanone. Some of the indanone-based chalcone derivatives have also been constructed based on the basic structure of Donepezil. These compounds have been studied mainly for its anti-Alzheimer activity and various other activities including anti-cancer, monoamine oxidase inhibitor, and the potential treatment of neurol..$^{4-8}$ The anti-inflammatory activity of the 6-hydroxyl-2-benzylidene-1-indanone was measured, and structure activities relationship of synthesised compounds was illustrated as presented in Figure 1..$^{9}$ In this study, ten indanone derivatives have been synthesised as potential DENV-2 NS2B/NS3 protease inhibitors.

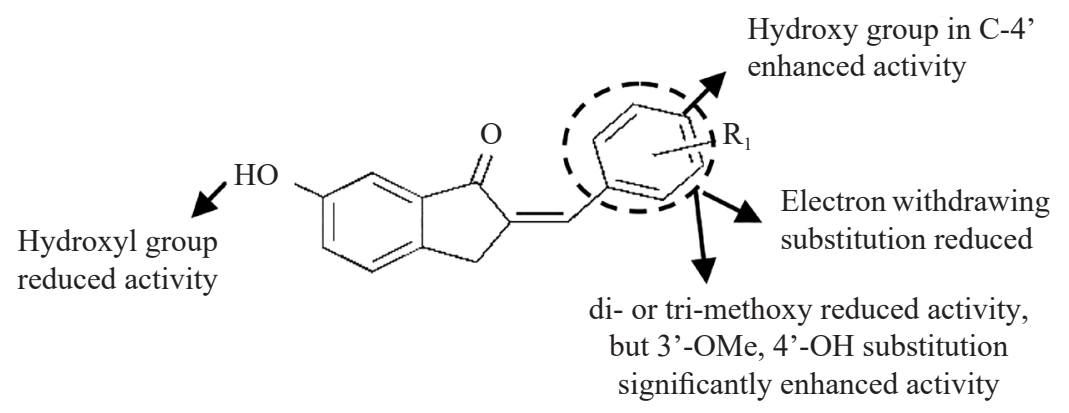

Figure 1: The structure activities relationship of 6-hydroxyl-2-benzylidene-1-indanone. 


\section{EXPERIMENTAL}

\subsection{The Synthesis of Benzylidene Indanone Derivatives}

Different types of aromatic aldehydes $(2.0 \mathrm{mmol}), 1 \mathrm{a}-1 \mathrm{j}$ were added into the stirred solution of indanone $(2.0 \mathrm{mmol}), 2$ in the presence of $10 \%$ of potassium hydroxide $(\mathrm{KOH})$ in methanol $(\mathrm{w} / \mathrm{v}) \cdot{ }^{10}$ The reaction of the benzylidene indanone derivatives, $3 \mathrm{a}-3 \mathrm{j}$ was depicted in Scheme 1. The reaction was monitored using thin-layer chromatography (TLC) with the solvent system of methanol:n-hexane (8.5:1.5 $(\mathrm{v} / \mathrm{v}))$ and the $\mathrm{pH}$ of the reaction mixture was then adjusted to $5-6$ by adding $10 \%$ hydrochloric acid solution $(\mathrm{w} / \mathrm{v})$. The precipitated was filtered using vacuum filtration, washed using cold methanol and dried under vacuum. The synthesized compounds were further recrystallised using a single-solvent recrystallization technique. After that, the synthesised compounds were further characterised to determine its physicochemical properties.

\subsection{Molecular Docking Studies}

The structure of the synthesised compounds was sketched using MarvinSketch 19.4.0 and converted into a 3D structure using HyperChem Pro 8.0 software. The molecular docking was performed using AutoDock 4.2 to identify the binding mode and conformation of the synthesised compound with the protease. ${ }^{11}$ The homologous crystal structure of protein generated by Wilchapong et al. were used. ${ }^{20}$ AutoDock Tools (ADT) version 1.5.6 was used in the preparation of docking simulation. The dimension of $74 \times 43 \times 64$ in $\mathrm{x}, \mathrm{y}, \mathrm{z}$ coordination were employed as the grid box size to cover the protease active site.

\section{RESULTS AND DISCUSSION}

The targeted compounds benzylidene indanone derivatives, $3 \mathrm{a}-3 \mathrm{j}$ as depicted in Scheme 1 were obtained by the aldol-type condensation. The yields of the synthesised compounds ranged from $40 \%$ to $80 \%$ after recrystallisation with ethanol. The data obtained were in agreement with the proposed structures. The Fourier transform infrared (FTIR) spectra revealed the appearance of aromatic $\mathrm{C}=\mathrm{C}$ band ranging at $1550-1650 \mathrm{~cm}^{-1}$ prove the formation of $\alpha, \beta$-unsaturated ketone. ${ }^{12,13}$ The ${ }^{1} \mathrm{H}$ NMR spectra showed the signals of respective proton according to their chemical shifts, multiplicities and coupling constants. The ${ }^{1} \mathrm{HNMR}$ spectra showed a singlet at 7.1-7.8 ppm corresponding to proton from the $-\mathrm{C}=\mathrm{CH}$ - of $\alpha, \beta$ unsaturated ketone bond resulting from the condensation of indanone and aromatic aldehyde. The ${ }^{13} \mathrm{C}$ NMR spectra of the synthesised compounds showed a signal at 
the range of 135-136 ppm corresponding to carbon-carbon double bond between the indanone and aromatic indicating the formation of the benzylidene indanone derivatives. ${ }^{14}$

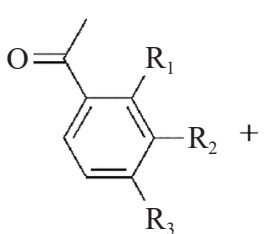

$1 \mathrm{a}-1 \mathrm{j}$<smiles>O=C1CCc2ccccc21</smiles>

2

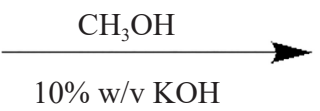

$10 \% \mathrm{w} / \mathrm{v} \mathrm{KOH}$

1a: $\mathrm{R}_{1}=\mathrm{H} ; \mathrm{R}_{2}=\mathrm{H} ; \mathrm{R}_{3}=\mathrm{H}$

1b: $\mathrm{R}_{1}=\mathrm{H} ; \mathrm{R}_{2}=\mathrm{H} ; \mathrm{R}_{3}=\mathrm{OCH}_{3}$

1c: $\mathrm{R}_{1}=\mathrm{H} ; \mathrm{R}_{2}=\mathrm{H} ; \mathrm{R}_{3}=\mathrm{CH}_{3}$

1d: $\mathrm{R}_{1}=\mathrm{H} ; \mathrm{R}_{2}=\mathrm{H} ; \mathrm{R}_{3}=\mathrm{Cl}$

1e: $\mathrm{R}_{1}=\mathrm{H} ; \mathrm{R}_{2}=\mathrm{H} ; \mathrm{R}_{3}=\mathrm{CH}\left(\mathrm{CH}_{3}\right)_{2}$

1f: $\mathrm{R}_{1}=\mathrm{H} ; \mathrm{R}_{2}=\mathrm{H} ; \mathrm{R}_{3}=\mathrm{N}\left(\mathrm{CH}_{3}\right)_{2}$

1g: $\mathrm{R}_{1}=\mathrm{H} ; \mathrm{R}_{2}=\mathrm{H} ; \mathrm{R}_{3}=\mathrm{NC}_{4} \mathrm{H}_{8} \mathrm{O}$

1h: $\mathrm{R}_{1}=\mathrm{H} ; \mathrm{R}_{2}=\mathrm{H} ; \mathrm{R}_{3}=\mathrm{NC}_{4} \mathrm{H}_{10}$

1i: $\mathrm{R}_{1}=\mathrm{OCH}_{3} ; \mathrm{R}_{2}=\mathrm{H} ; \mathrm{R}_{3}=\mathrm{OCH}_{3}$

1j: $\mathrm{R}_{1}=\mathrm{H} ; \mathrm{R}_{2}=\mathrm{NO}_{2} ; \mathrm{R}_{3}=\mathrm{OCH}_{3}$

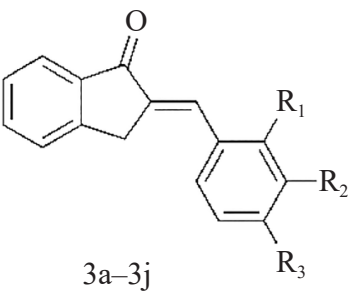

$3 a-3 j$

Scheme 1: The synthesis of benzylidene indanone derivatives.

The in-silico studies were conducted to screen the compounds with molecular interaction towards DENV-2 NS2B/NS3 protease, as it plays an important role in virus replication. ${ }^{15}$ The three catalytic triad amino acid residues were located in NS3 serine protease namely His51, Asp75 and Ser135. The NS2B protease acted as a NS3 serine protease co-factor for an optimal catalytic activity. ${ }^{16,17}$ The DENV-2 NS2B/NS3 protease was reported to have an activity by cleaving the adjacent basic residues of NS2A/NS2B, NS2B/NS3, NS3/NS4A and NS4B/NS5 junction. ${ }^{18,19}$ The homology protein crystal structure generated by Wilchapong et al. was used due to the lack of DENV-2 NS2B/NS3 serine protease inhibitorbound $3 \mathrm{D}$ structures and some of the protease database structures were found to have a missing amino acid residues. ${ }^{20}$

The Lamarckian genetic algorithm was employed in AutoDock 4.2 to determine the binding modes and synthesised compounds conformation towards DENV-2 NS2B/NS3 protease homology protein crystal structure. The covalent map parameter was used during positive control docking simulation to constrain the molecular geometry of the peptide inhibitor. After that, compounds $3 a-3 j$ were docked with Wilchapong homology crystal structure using the same parameter. 
The free energy of binding (FEB) obtained was tabulated in Table 1. The compounds, $3 \mathrm{a}-3 \mathrm{j}$ fit into the allosteric pocket of NS2B/NS3 serine protease (Figure 2). The compounds $3 \mathrm{~g}$ and $3 \mathrm{~h}$ that have the lowest free energy of binding of $-7.3 \mathrm{kcal} \mathrm{mol}^{-1}$ showed a higher binding affinity towards the amino acid residues in the allosteric pocket.

Table 1: The free energy of binding

\begin{tabular}{cc}
\hline Compound & FEB $\left(\mathrm{kcal} \mathrm{mol}^{-1}\right)$ \\
\hline $3 \mathrm{a}$ & -6.4 \\
$3 \mathrm{~b}$ & -6.4 \\
$3 \mathrm{c}$ & -6.7 \\
$3 \mathrm{~d}$ & -6.7 \\
$3 \mathrm{e}$ & -6.7 \\
$3 \mathrm{f}$ & -6.7 \\
$3 \mathrm{~g}$ & -7.3 \\
$3 \mathrm{~h}$ & -7.3 \\
$3 \mathrm{i}$ & -6.7 \\
$3 \mathrm{j}$ & -6.8 \\
\hline
\end{tabular}

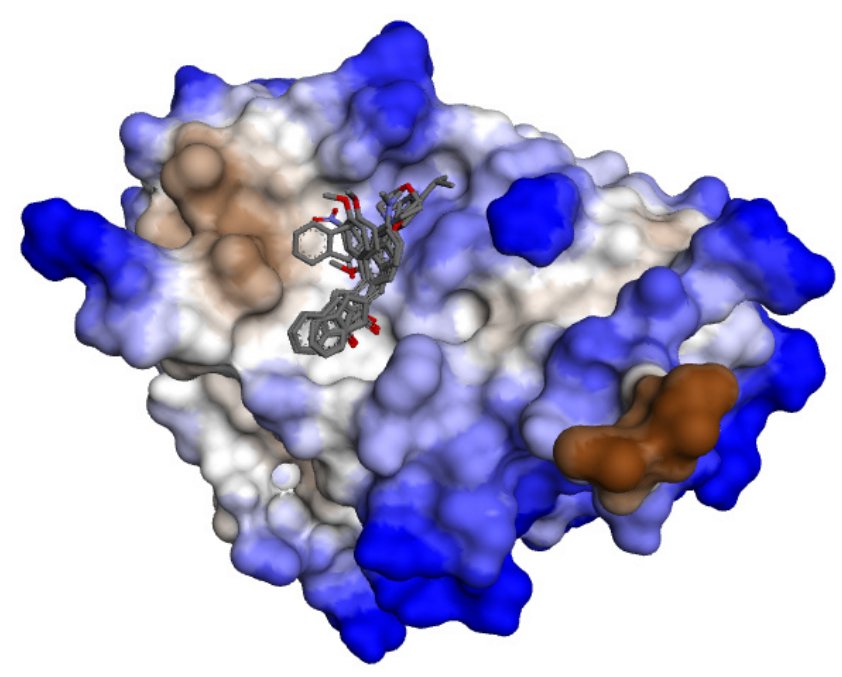

Figure 2: The binding conformation of the synthesised compounds $(3 \mathrm{a}-3 \mathrm{j})$.

The detailed binding interaction of the compound $3 \mathrm{~g}$ and $3 \mathrm{~h}$ with the amino acid residues were shown in Figure 3. Both compounds showed one hydrogen bonding interaction, two $\pi-\pi$ stacking interaction, and one $\pi$-alkyl interaction with the amino acid residue in the DENV-2 NS2B/NS3 protease. 
The presence of the aromatic group in the synthesised benzylidene indanone derivatives enhance the possibilities of van der Waals interaction. The $\pi$ - $\pi$ stacking interaction between the His51 and the benzylidene moiety in compounds $3 \mathrm{~g}$ and $3 \mathrm{~h}$ suggested affecting DENV-2 NS2B/NS3 replication as His51 is one of the catalytic triads of NS2B required for replication activities. ${ }^{21}$ Besides that, the indanone moiety in the synthesised compounds contributed to another $\pi$ - $\pi$ stacking interaction between the aromatic ring moieties present in the Tyr 161.

The hydrogen bond interaction of compound $3 \mathrm{~g}$ and amino acid residue Gly82 occurred at the morpholine ring with a distance of $3.56 \AA$, as the hydrogen located at the carbon next to the oxygen act as the hydrogen donor. Whilst, the hydrogen bond interaction of $3.47 \AA$ between $3 \mathrm{~h}$ and amino acid residue Asn 154 exist at the nitrogen atom in the piperidinyl moiety. The stability of the hydrogen bond network between the synthesised compounds and the amino acid residues dominates the binding strength and therefore affecting the rate of inhibitors. One of the criteria of a good hydrogen bond interaction is the distance between hydrogen donor and acceptor was shorter than $3.5 \AA .{ }^{22}$ Hence, the hydrogen bond presence of compound $3 \mathrm{~h}$ was suggested to be stronger as it is in a shorter distance than compound $3 \mathrm{~g}$.
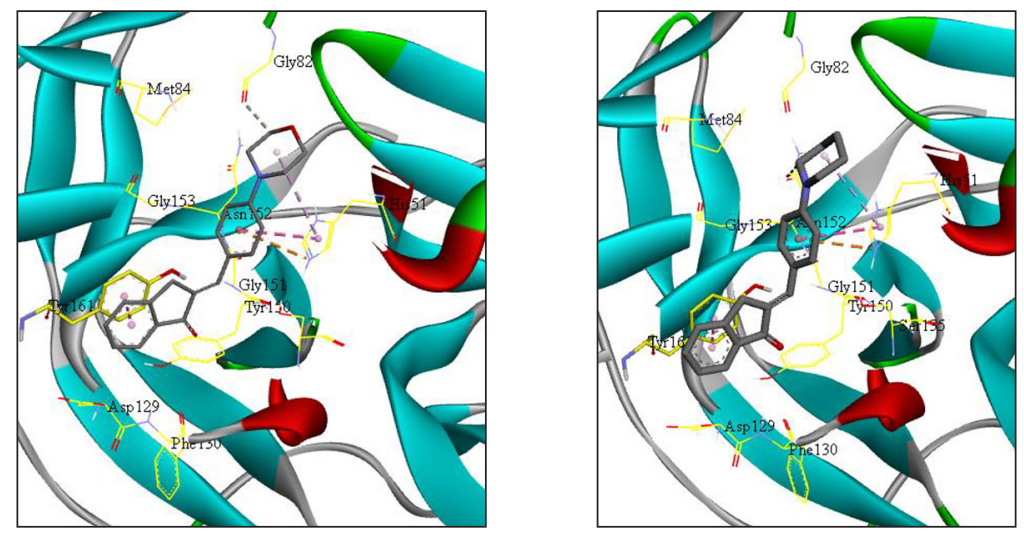

Figure 3: The binding interaction of compounds $3 g$ and $3 \mathrm{~h}$ (- hydrogen bond; $-\pi$-cation; $-\pi$ - $\pi$ stacking; - $\pi$-alkyl)

\section{CONCLUSION}

The significant molecular interaction between the synthesised compounds and DENV2 NS2B/NS3 serine protease were revealed by molecular studies indicating their potential activity as a protease inhibitor. The compound $3 \mathrm{~g}$ is predicted to have a higher DENV-2 NS2B/NS3 protease inhibitor as it has a good hydrogen 
bond interaction with a significant van der Waals force between the synthesised compound and the amino acid residues. Further verification studies such as, invitro, toxicity analysis, and in-vivo is required for identifying the possibilities of the indanone derivatives to be a lead molecule for anti-dengue drug development.

\section{ACKNOWLEDGEMENTS}

The authors thanked the Malaysian Government, French Government, University of Lorraine, and Universiti Sains Malaysia for the financial support of this study through the Research University grant, RUI 1001/PKIMIA/8011072

\section{REFERENCES}

1. World Health Organization (WHO). (2019). Dengue and severe dengue. Retrieved 15 September 2019 from https://www.who.int/news-room/fact-sheets/detail/ dengue-and-severe-dengue.

2. Guy, B. et al. (2011). From research to phase III: Preclinical, industrial and clinical development of the Sanofi Pasteur tetravalent dengue vaccine. Vac., 29(42), 72297241, https://doi.org/10.1016/j.vaccine.2011.06.094.

3. WHO. (2018). Dengue and severe dengue. Retrieved 7 April 2019 from https://www.who.int/en/news-room/fact-sheets/detail/dengue-and-severe-dengue.

4. Cho, Y. S. et al. (2011). Inhibition of acetylcholinesterase by gallic acid-graftedchitosans. Carbohydr. Polym., 84(1), 690-693, https://doi.org/10.1016/j. carbpol.2010.12.040.

5. Patil, S. A., Patil, R. \& Patil, S. A. (2017). Recent developments in biological activities of indanones. Eur. J. Med. Chem., 138, 182-198, https://doi.org/10.1016/j. ejmech.2017.06.032.

6. Saxena, H. O. et al. (2008). Gallic acid-based indanone derivatives as anticancer agents. Bioorg. Med. Chem. Lett., 18(14), 3914-3918, https://doi.org/10.1016/j. bmcl.2008.06.039.

7. Nel, M. S. et al. (2016). 2-heteroarylidene-1-indanone derivatives as inhibitors of monoamine oxidase. Bioorg. Chem., 69, 20-28, https://doi.org/10.1016/j. bioorg.2016.09.004.

8. Janse van Rensburg, H. D. et al. (2019). Methoxy substituted 2-benzylidene-1indanone derivatives as $\mathrm{A} 1 \mathrm{and} / \mathrm{or} \mathrm{A} 2 \mathrm{~A} \mathrm{AR}$ antagonists for the potential treatment of neurological conditions. MedChemComm., 10(2), 300-309, https://doi. org $/ 10.1039 / \mathrm{c} 8 \mathrm{md} 00540 \mathrm{k}$.

9. Xiao, S. et al. (2018). Design, synthesis, and structure-activity relationships of 2-benzylidene-1-indanone derivatives as anti-inflammatory agents for treatment of acute lung injury. Drug Des. Dev. Ther., 12, 887-899, https://doi.org/10.2147/ DDDT.S160314. 
10. Nielsen, A. T. \& Houlihan, W. J. (2011). The aldol condensation. New York: John Wiley \& Sons.

11. Morris, G. M. et al. (2009). AutoDock4 and AutoDockTools4: Automated docking with selective receptor flexibility. J. Comp. Sci., 30(16), 2785-2791, https://doi. org/10.1002/jcc.21256.

12. Mohd Sabri, M. S., Oo, C. W. \& Yam, M. F. (2018). Synthesis, characterization and vasolidation properties of indanone-based chalcones. J. Phys. Sci., 29(1),99106, https://doi.org/10.21315/jps2018.29.s1.13.

13. Coates, J. (2007). Interpretation of infrared spectra: A practical approach. In Meyers, R. A. (Ed.), Encyclopedia of analytical chemistry. New York: John Wiley \& Sons,1-23, https://doi.org/10.1002/9780470027318.a5606.

14. Wachter, N. (2013). Using NMR to investigate products of aldol reactions: Identifying aldol addition versus condensation products or conjugate addition products from crossed aldol reactions of aromatic aldehydes and ketones. ACS Symp. Ser., 1128, 91-102, https://doi.org/10.1021/bk-2013-1128.ch007.

15. Othman, R. et al. (2008). Docking of noncompetitive inhibitors into dengue virus Type 2 protease: Understanding the interactions with allosteric binding sites. J. Chem. Inf. Model., 48(8), 1582-1591, https://doi.org/10.1021/ci700388k.

16. Arias, C. F., Preugschat, F. \& Strauss, J. H. (1993). Dengue 2 virus NS2B and NS3 form a stable complex that can cleave NS3 within the helicase domain. Virol., 193(2), 888-899, https://doi.org/10.1006/viro.1993.1198.

17. Preugschat, F., Yao, C. W. \& Strauss, J. H. (1990). In vitro processing of dengue virus type 2 nonstructural proteins NS2A, NS2B, and NS3. J. Virol., 64(9), 43644374, https://www.ncbi.nlm.nih.gov/pubmed/2143543.

18. Cahour, A., Falgout, B. \& Lai, C. J. (1992). Cleavage of the dengue virus polyprotein at the NS3/NS4A and NS4B/NS5 junctions is mediated by viral protease NS2BNS3, whereas NS4A/NS4B may be processed by a cellular protease. J. Virol., 66(3), 1535-1542.

19. Falgout, B. et al. (1991). Both nonstructural proteins NS2B and NS3 are required for the proteolytic processing of dengue virus nonstructural proteins. J. Virol., 65(5), 2467-2475.

20. Wilchapong, K., Pianwanit, S. \& Sippl, W. (2010). Homology modeling and molecular dynamics simulations of dengue virus NS2B/NS3 protease: Insight into molecular interaction. J. Mol. Recog., 23, 283-300, https://doi.org/10.1002/ jmr.977.

21. Noble, C. G. et al. (2012). Ligand-bound structures of the dengue virus protease reveal the active conformation. J. Virol., 86(1), 438-446, https://doi.org/10.1128/ JVI.06225-11.

22. Li, D. et al. (2011). Strength of hydrogen bond network takes crucial roles in the dissociation process of inhibitors from the hiv-1 protease binding pocket. PLoS ONE, 6(4), https://doi.org/10.1371/journal.pone.0019268 